\section{(1)}

CrossMark

\title{
The supportive care needs of people living with pulmonary fibrosis and their caregivers: a systematic review
}

\author{
Joanna Y.T. Lee (1) ${ }^{1,2,3}$, Gabriella Tikellis ${ }^{1,2,3}$, Tamera J. Corte $3,4,5$, \\ Nicole S. Goh ${ }^{6,7,8}$, Gregory J. Keir ${ }^{9}$, Lissa Spencer ${ }^{4,10}$, Debra Sandford ${ }^{11,12}$, \\ Yet H. Khor (10) $6,7,8,13$, Ian Glaspole (10) $2,3,8$, John Price ${ }^{14}$, \\ Alison J. Hey-Cunningham ${ }^{3,4}$, Jamie Maloney ${ }^{15}$, Alan K.Y. Teoh ${ }^{3,4,5}$, \\ Alice L. Watson ${ }^{1}$ and Anne E. Holland (1) ${ }^{1,2,3,7,8}$
}

\begin{abstract}
Affiliations: ${ }^{1}$ Physiotherapy, La Trobe University, Melbourne, Australia. ${ }^{2}$ Allergy, Immunology and Respiratory Medicine, Monash University, Melbourne, Australia. ${ }^{3}$ NHMRC Centre of Research Excellence in Pulmonary Fibrosis, Sydney, Australia. ${ }^{4}$ The University of Sydney, Sydney, Australia. ${ }^{5}$ Respiratory Medicine, Royal Prince Alfred Hospital, Camperdown, Australia. ${ }^{6}$ Austin Health, Heidelberg, Australia. ${ }^{7}$ Institute for Breathing and Sleep, Melbourne, Australia. ${ }^{8}$ Alfred Health, Melbourne, Australia. ${ }^{9}$ Princess Alexandra Hospital, Woolloongabba, Australia. ${ }^{10}$ Royal Prince Alfred Hospital, Camperdown, Australia. ${ }^{11}$ Royal Adelaide Hospital, Adelaide, Australia. ${ }^{12}$ The University of Adelaide, Adelaide, Australia. ${ }^{13}$ University of Melbourne, Melbourne, Australia. ${ }^{14}$ Institute for Respiratory Health, Nedlands, Australia. ${ }^{15}$ Lung Foundation Australia, Brisbane, Australia.
\end{abstract}

Correspondence: Anne E. Holland, Physiotherapy Dept, Alfred Hospital, Commercial Rd, Melbourne, 3004 Australia. E-mail: a.hollanddalfred.org.au

@ERSpublications

Summary of unmet care needs reported by people with pulmonary fibrosis and their caregivers across a range of settings and countries. People with pulmonary fibrosis and their caregivers have many unmet needs. The results can help improve care provided for people with pulmonary fibrosis and caregivers. http://bit.ly/39PdjfQ

Cite this article as: Lee JYT, Tikellis G, Corte TJ, et al. The supportive care needs of people living with pulmonary fibrosis and their caregivers: a systematic review. Eur Respir Rev 2020; 29: 190125 [https://doi. org/10.1183/16000617.0125-2019].

\section{ABSTRACT}

Background: People with pulmonary fibrosis often experience a protracted time to diagnosis, high symptom burden and limited disease information. This review aimed to identify the supportive care needs reported by people with pulmonary fibrosis and their caregivers.

Methods: A systematic review was conducted according to PRISMA guidelines. Studies that investigated the supportive care needs of people with pulmonary fibrosis or their caregivers were included. Supportive care needs were extracted and mapped to eight pre-specified domains using a framework synthesis method.

Results: A total of 35 studies were included. The most frequently reported needs were in the domain of information/education, including information on supplemental oxygen, disease progression and prognosis, pharmacological treatments and end-of-life planning. Psychosocial/emotional needs were also frequently reported, including management of anxiety, anger, sadness and fear. An additional domain of "access to care" was identified that had not been specified a priori; this included access to peer support, psychological support, specialist centres and support for families of people with pulmonary fibrosis.

Conclusion: People with pulmonary fibrosis report many unmet needs for supportive care, particularly related to insufficient information and lack of psychosocial support. These data can inform the development of comprehensive care models for people with pulmonary fibrosis and their loved ones.

This article has supplementary material available from err.ersjournals.com

This study is registered at PROSPERO with registration number CRD42019131878.

Provenance: Submitted article, peer reviewed

Received: 24 Sept 2019 | Accepted after revision: 03 Jan 2020

Copyright $\odot$ ERS 2020. This article is open access and distributed under the terms of the Creative Commons Attribution Non-Commercial Licence 4.0. 


\section{Introduction}

People living with pulmonary fibrosis often experience a protracted route to diagnosis, high symptom burden, limited disease information and anxiety about the future [1]. While antifibrotic treatments have brought a sense of hope that disease progression can be slowed, these treatments may not relieve symptoms or improve quality of life, and some patients do not meet the criteria for funded treatment. In a recent study, half of the patients with pulmonary fibrosis reported four or more unmanaged symptoms such as breathlessness, depression, cough, sleep difficulty, anxiety and fatigue and perceived few options for symptom control $[2,3]$. Breathlessness and cough are most prevalent, occurring in 54-98\% and 59-100\% of people with pulmonary fibrosis, respectively [4]. Anxiety is present in up to one-third of individuals living with pulmonary fibrosis, while symptoms of depression occur in $25 \%$ [5].

Supportive care needs can be defined as informational, emotional, spiritual, social or physical needs experienced at any stage of the healthcare journey [6]. A number of studies have investigated the supportive care needs of people with pulmonary fibrosis in settings across the world [16, 20, 25, 29]. Studies conducted prior to the antifibrotic era (pre-2011) tended to focus on the experience of diagnosis [1, 17] with limited data on the support needs at other stages of the pulmonary fibrosis journey. Since then, there have been emerging data documenting the experience of modern pulmonary fibrosis care in settings across the world, providing more information about experiences with new treatments [7, 8]. All these studies provide valuable insights that could enhance patient-centred care and improve outcomes. One previous systematic review synthesised the literature on symptom prevalence in people with pulmonary fibrosis [4]; however, only quantitative studies were included and other supportive care needs were not addressed. The aim of this review was to identify the supportive care needs reported by people living with pulmonary fibrosis and their caregivers.

\section{Methods}

A systematic review was conducted of studies reporting the supportive care needs of people with pulmonary fibrosis and their caregivers. The review was registered at PROSPERO (CRD42019131878). Four electronic databases (EMBASE, CINAHL, MEDLINE and PsychINFO) were searched from their inception to 10 July 2019 (table S1). The search was limited to publications in English and studies that included adults aged $\geqslant 18$ years. There was no restriction on year of publication, type of literature, study methodology or research design. Studies were included if they investigated the supportive care needs of people with pulmonary fibrosis or their caregivers. Studies reporting the supportive care needs of people with other types of lung disease were excluded, unless results were presented separately for people with pulmonary fibrosis. We also excluded studies that tested the psychometric properties of tools designed to measure supportive care needs. Reporting was performed according to the PRISMA (Preferred Reporting Items for Systematic Reviews and Meta-Analyses) guidelines [9].

Two independent researchers (A. Watson and J. Lee) screened the titles and abstracts of retrieved studies for eligibility. Those that met the inclusion criteria, or where it was unclear whether the study met the inclusion criteria, were retrieved in full. Additional searches were conducted on Scopus and Google Scholar to identify published articles that were linked to any retrieved conference abstracts. All full texts were reviewed by two independent researchers (A.E. Holland and J. Lee) to determine study inclusion, with any disagreements resolved by consensus. The reference lists of all included studies were searched for additional studies that met the inclusion criteria.

Two researchers ( J. Lee and G. Tikellis) independently assessed the methodological quality of included studies using the QualSyst tool, which is used to evaluate the quality of both qualitative and quantitative studies [10]. In this tool, the qualitative scale has 10 items with a total possible sum of 20. Each item is scored according to the extent to which the criteria was met (yes: 2, partial: 1, no: 0).A summary score was recorded for each study. Summary scores for quantitative studies were similarly calculated based on 14 items with a maximum possible sum of 28 . Five items could be marked as "not applicable" and excluded from the calculation of the summary score, where applicable. Where studies used mixed methods, assessment was performed according to the more dominant method. Any scoring discrepancies between the researchers were resolved by discussion until consensus was reached. No studies were excluded based on methodological quality.

Data extraction was performed by one reviewer ( J. Lee), with random checks for accuracy carried out by a second reviewer (A. Hey-Cunningham), using a data extraction form specifically developed for this study and piloted on two randomly selected studies. Data extracted included study design, characteristics of the sample, methods of data collection and analysis, and the supportive care needs identified. Supportive care needs were classified into eight conceptual domains as previously defined [11]: 1) Physical/cognitive: symptom management and treatment-related toxicity, and cognitive dysfunction; 2) Psychosocial/ emotional: psychological/emotional symptoms such as depressive mood, anxiety, fear/worry and despair; 
3) Family related: dysfunctional family relationships and fears/concerns for family's future; 4) Social/ societal: experience of social isolation, inefficient social support and diminished socialisation; 5) Interpersonal/intimacy: altered body image or sexuality, sexual health problems and compromised intimacy with partner; 6) Practical/daily living: transportation, advanced directives, out-of-hours accessibility, funeral care, financial strain, experience of restriction in daily living tasks such as housekeeping, and exercise; 7) Information/education: lack of information, uncertainty about diagnosis/ treatment and uncertainty/lack of knowledge about self-care; 8) Health system/patient-clinician communication: insufficient communication between patients and clinicians, satisfaction with care, participation in decision-making and preferences in communication.

Individual supportive care needs were extracted and mapped to the relevant domain using a framework synthesis method, with supportive quotes extracted verbatim from each paper. Thematic analysis was conducted to identify any new domains for data outside the pre-specified domains. The prevalence of each supportive care need was reported where such information was available from quantitative studies. The frequency of studies identifying the different domains was reported. As the majority of studies were qualitative in nature, a narrative synthesis was performed and representative quotes presented. Where there were sufficient data, subgroup analyses were performed for: 1) idiopathic pulmonary fibrosis (IPF) versus other types of pulmonary fibrosis; 2) antifibrotic era versus pre-antifibrotic era; and 3) disease stage, i.e. time of diagnosis versus routine follow-up versus end of life.

\section{Results}

A total of 4180 records were identified after excluding duplicates, with 83 studies retrieved for full-text review (figure 1). A total of 35 studies were included, consisting of 28 full-text articles [1, 3, 7, 8, 12-35] and seven conference abstracts [36-42]. These had been published between 2005 and 2019 and were predominantly conducted in the USA $(n=13)$, UK $(n=10)$ and other parts of Europe $(n=8)$. Overall, 2621 (69\%) patients, 590 (16\%) informal caregivers and 558 (15\%) healthcare professionals/clinical researchers/ policy experts were included. One study did not report number of participants (i.e. patients and staff) [37], while another study reported the responses $(n=471)$ received via blog entries or discussion forum threads on three interactive pulmonary fibrosis-focused websites, rather than the number of participants [12]. The characteristics of included studies are presented in table S2.

Studies most commonly included participants with IPF and their caregivers ( $n=3076,96 \%)$; in 12 studies a small number of people with other pulmonary fibrosis diagnoses were also included (e.g. nonspecific interstitial pneumonia, chronic hypersensitivity pneumonitis and connective tissue disease). 11 studies reported pulmonary function, with forced vital capacity $31-125 \%$ pred $(n=7)$ and diffusing capacity of the lung for carbon monoxide $13-95 \%$ pred $(n=7)$. About half of the studies recruited participants from specialist clinics $(n=19)$, other settings included patient support groups $(n=4)$, patient advocacy organisations $(n=3)$, tertiary hospitals $(n=2)$, a non-profit organisation $(n=1)$, an IPF registry $(n=1)$ and a lung transplant centre $(\mathrm{n}=1) .17(47 \%)$ studies were conducted in the antifibrotic era $[7,8,14,16,20-24$, 30-35, 41, 42]. Most studies reported experiences of diagnosis and treatment, with six focused specifically on end-of-life or palliative care $[3,20,25,32,37,40]$.

15 studies used semi-structured and in-depth interviews (43\%), 11 administered questionnaires [7, 14, 17, $24,33,37-42]$, five used focus groups [13, 20, 21, 23, 27], three used a combination of methods [26, 31, 35], and one used narrative data from websites [12]. 23 (65\%) studies used a qualitative data analysis method. Quantitative methods were used to report findings regarding disease management [7, 38, 39], patient education and support [17,24], patient expectations of care [41], quality of life [26, 38, 41] and anxiety [24]. Six studies were conducted using mixed methods [26, 31, 33, 35, 37, 42]. Meta-analysis was not possible as the number of quantitative studies was small and the data heterogeneous.

Quality assessment scores generally ranged from 70-95\% (tables S3 and S4). However, four studies, all reported as abstracts only, scored $\leqslant 55 \%$. Strengths of the qualitative studies were clearly described research questions or objectives, appropriate and easily identified research designs, and study conclusions that were supported by data. Most qualitative studies scored poorly on reporting evidence of reflexivity, the connection to a theoretical framework and sampling strategy (frequently a convenience sample). Quantitative studies scored well on items related to clearly specifying objectives and conclusions supported by study results, but reporting of participant characteristics was frequently poor.

\section{Supportive care needs}

The supportive care needs of people with pulmonary fibrosis are summarised in tables 1-4. The most frequently reported needs were in the domain of information/education (26 studies) and psychosocial/ emotional care (22 studies), although all domains were represented. An additional domain of "access to care" was identified, which was reported in 27 studies. 

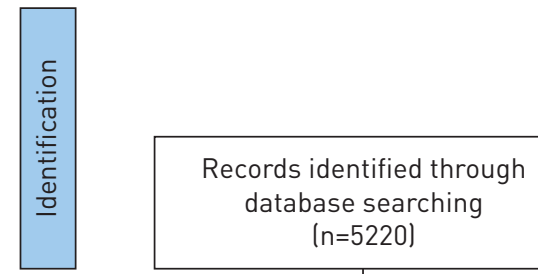

Additional records identified through other sources $(n=5)$

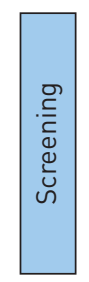

Records after duplicates removed $(n=4180)$
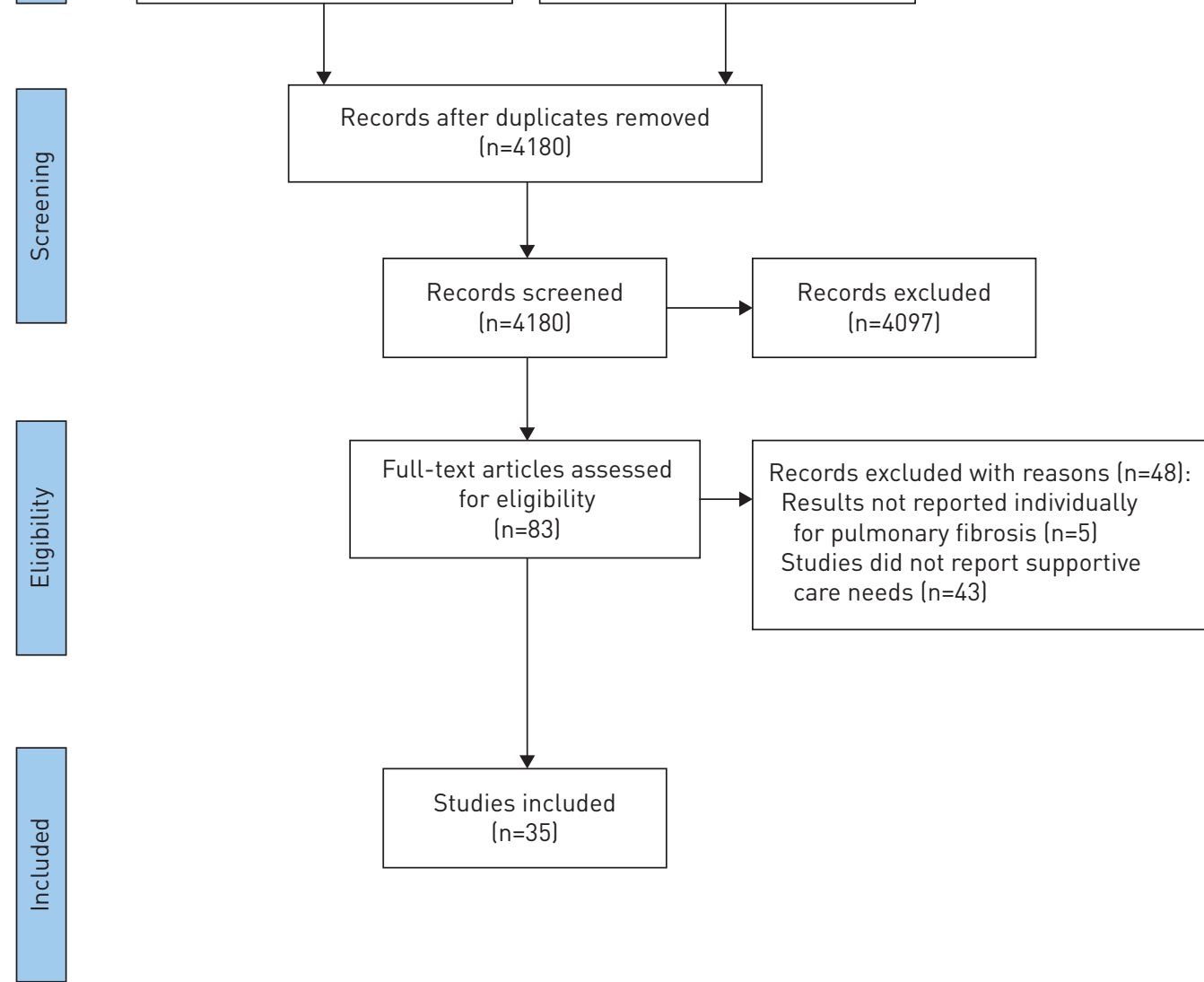

FIGURE 1 PRISMA flow diagram.

\section{Physical/cognitive needs}

The most common and distressing symptoms were cough, dyspnoea and fatigue, which were reported in 16 studies (table 1). Paroxysms of cough were exhausting for patients and impacted on sleep quality for both patients and caregivers $[3,20,22]$. Patients reported having to take breaks during simple tasks such as showering or bending in order to catch their breath [27]. Cough-related incontinence was also reported [28], which caused significant distress in $15 \%$ of patients (95\% CI 7-23\%) [39]. Patients often experienced side-effects of antifibrotic treatment including weight loss, nausea, diarrhoea and photosensitivity, and desired help to better manage these $[8,16] .22 \%$ of patients with pulmonary fibrosis felt their symptoms were not controlled (95\% CI 13-30\%) [39].

\section{Psychosocial/emotional needs}

The psychosocial/emotional needs of patients and caregivers were reported in 22 studies (table 1). At the time of diagnosis patients and caregivers often experienced anger, frustration and loss of control $[13,23,25]$. Twothirds of patients and caregivers experienced anxiety [24]. They were fearful about disease progression and struggled with uncertainty about the future, including loss of employment or income $[8,21,30]$. Patients worried about losing independence and becoming a burden on their family and society [1, 20, 27], while caregivers feared losing their loved ones [13]. Many patients felt guilty for not being able to fulfil their usual roles [28]. Sadness and depression were frequently reported [1, 8, 13, 19, 21, 22, 27]. In a European survey, $80 \%$ of patients wanted the possibility of psychological support and $23 \%$ thought it was lacking in current care [24].

As the disease progressed, both patients and caregivers felt overwhelmed and helpless [3, 13]. Some patients experienced panic due to shortness of breath [3]. Being prescribed supplemental oxygen was often 
TABLE 1 Summary of supportive care needs: physical/cognitive and psychosocial/emotional domains

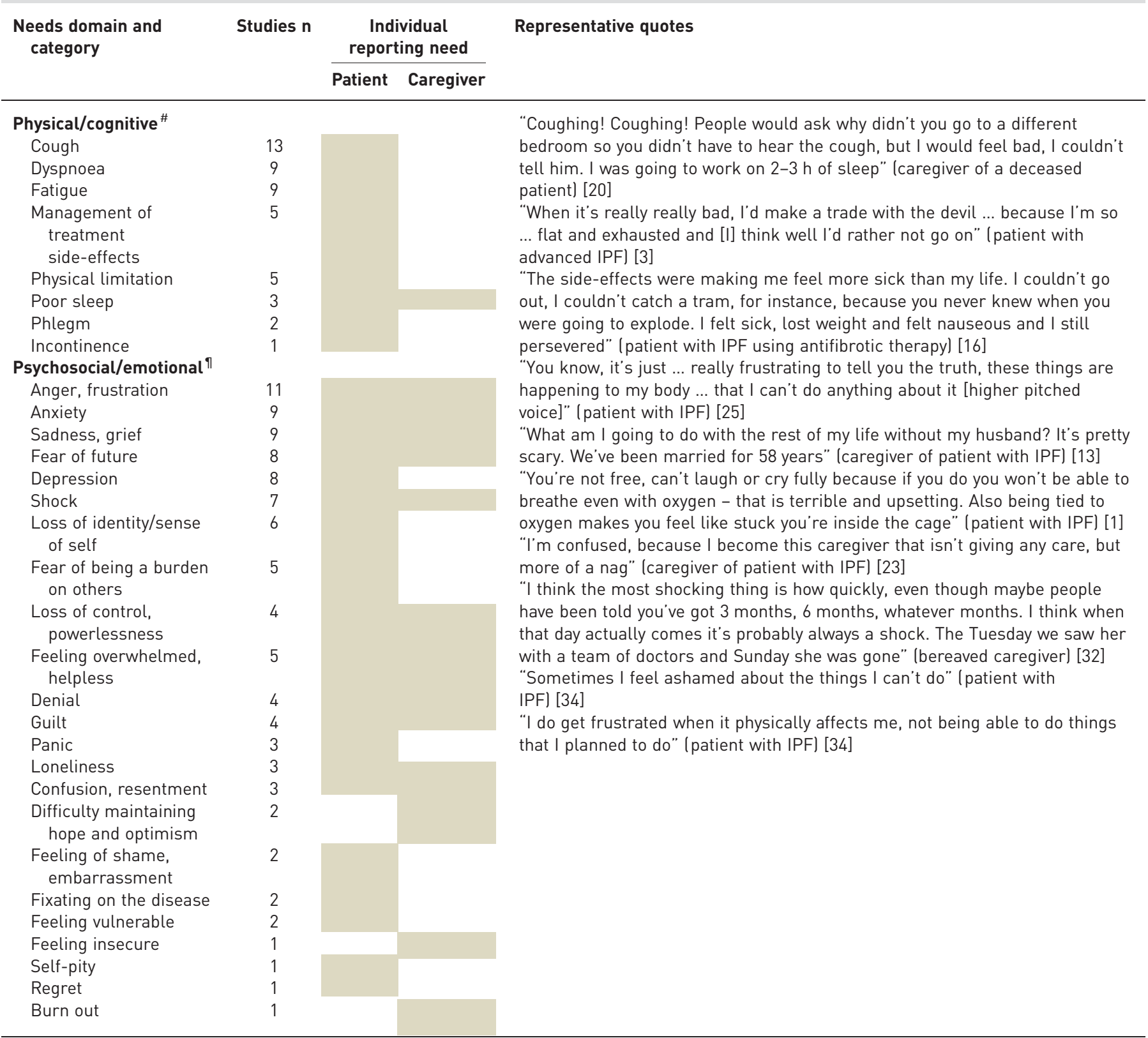

Shading indicates where supportive care need was identified by the patient and/or caregiver. IPF: idiopathic pulmonary fibrosis. ${ }^{\#}: \mathrm{n}=16$; ${ }^{1}: \mathrm{n}=22$.

viewed as "losing the battle" [19] and was associated with a loss of hope [8]. Patients felt vulnerable using oxygen, and ashamed or embarrassed because it made their illness visible to others $[8,15]$. Many patients and caregivers felt lonely and isolated as the disease took over their lives [3, 22]. Some caregivers found it difficult to adapt to their new roles, some felt resentful, some were conflicted between their duty and living their own lives, and some burnt out [13]. More than $60 \%$ of the partners of people with pulmonary fibrosis wished for more care, specifically for caregivers [24].

\section{Family-related needs}

The family-related needs of patients and caregivers were reported in 14 studies (table 2). Losing independence and becoming a burden on the family were prominent concerns for patients [15], with $27 \%$ reporting that they worried about the effect of their illness on their loved ones [39]. Family caregivers assisted with personal hygiene and supplemental oxygen use; many described how increased reliance on their spouse had caused relationship strain. Family members often found it difficult to maintain 
TABLE 2 Summary of supportive care needs: family-related, social/societal and interpersonal/intimacy domains

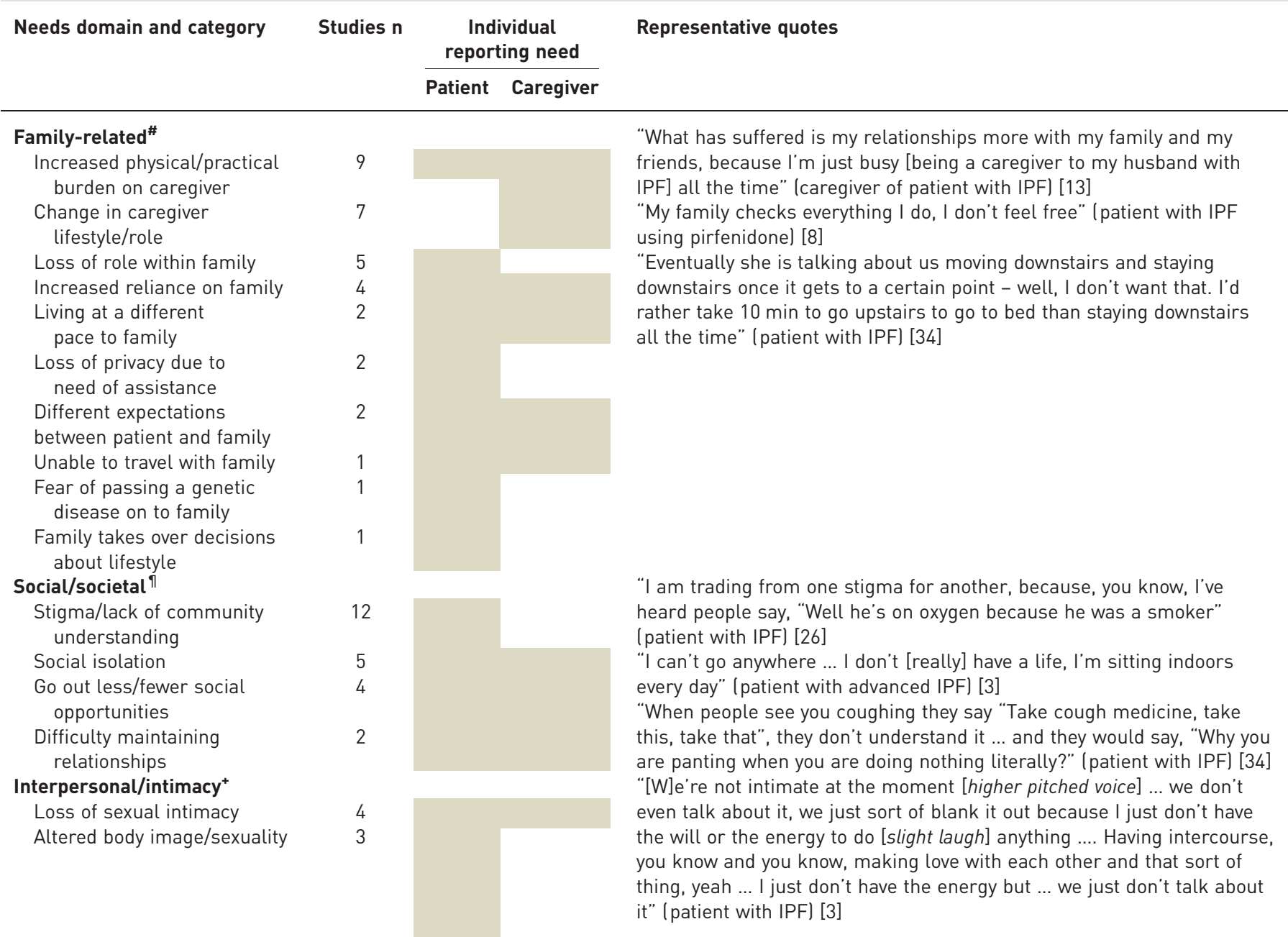

Shading indicate where supportive care need was identified by the patient and/or caregiver. IPF: idiopathic pulmonary fibrosis. ${ }^{\#}: \mathrm{n}=14 ;{ }^{\text {? }}: \mathrm{n}=17$; ${ }^{+}: \mathrm{n}=4$.

relationships with others outside the family as caregiver responsibilities consumed most of their time [3]. Many patients noted a loss of privacy because they needed assistance from the family with so many tasks [27]. Some patients feared passing on the disease to their families [15]. Patients and families were often living at a different pace; some felt frustrated that their loved ones "can't keep up", while many experienced difficulties in travelling together [19].

\section{Social/societal needs}

The social/societal needs of patients and caregivers were reported in 17 studies (table 2). Patients frequently reported social stigma and a lack of community understanding of pulmonary fibrosis $[15,19,26]$, with over one-third of patients and partners regularly feeling misunderstood because people did not know what pulmonary fibrosis was [24]. This made it difficult to interact with others, including friends, family, employers and insurers $[18,21,24]$. Patients believed that an increased disease awareness among healthcare professionals was needed to improve accurate and timely diagnosis [1]. Both patients and caregivers experience social isolation $[1,3,22]$. Patients reduced their attendance at social events as the disease progressed, while caregivers often gave up their own social life [3, 13]. Many patients were reluctant to attend social occasions that involve crowds of people to avoid "catching something" [27].

\section{Interpersonal/intimacy needs}

Th interpersonal/intimacy needs of patients and caregivers were reported in four studies (table 2). Both patients and spouses expressed decreased libido and reduced physical stamina during sexual intercourse [3, 27]. 
TABLE 3 Summary of supportive care needs: practical/daily living and information/education domains

\begin{tabular}{|c|c|c|c|}
\hline \multirow[t]{2}{*}{ Needs domain and category } & \multirow[t]{2}{*}{ Studies $\mathbf{n}$} & $\begin{array}{l}\text { Individual } \\
\text { reporting need }\end{array}$ & \multirow[t]{2}{*}{ Representative quotes } \\
\hline & & Caregiver & \\
\hline
\end{tabular}

\section{Practical/daily living ${ }^{\#}$}

Loss of independence/decreased ability to perform activities of daily living

Financial burden/loss of income

End-of-life planning linsufficient information/do not have a plan)

Requiring more time, planning and adaptation both inside and outside of home

Physical challenges of transporting oxygen

Given up hobbies

Concerns with oxygen limitation outside of home

Physical challenges of using oxygen at home

\section{Information/education"}

Disease progression and prognosis

Supplemental oxygen, including

travel with oxygen

Pharmacological treatments

Planning for end-of-life

Coping strategies

Managing symptoms (breathlessness and cough)

Non-pharmacological treatments/ alternative therapies

Research and clinical trials

Pathophysiology

How to provide practical/emotional care for loved one with pulmonary fibrosis

Lung transplantation

Understanding tests used for diagnosis and monitoring

Treatment centres/referral to suitable specialists

Travelling with pulmonary fibrosis

How to recognise and deal with important signs and symptoms

Signs and symptoms of pulmonary fibrosis

Causes of pulmonary fibrosis

Managing comorbidities

Success stories of living with pulmonary fibrosis

Homecare/drug delivery

Advocacy

How to avoid infection

How to communicate with a frustrated/ angry/depressed patient

How doctors follow IPF over time

How to access community supports

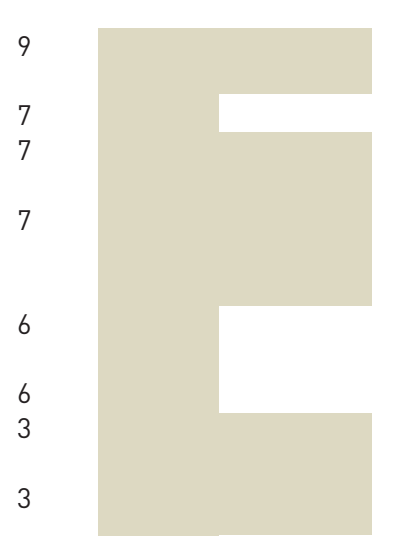

“He can't bend over. If he bends over, forget it. So I empty out the bottom of the dishwasher. He empties the top, because he likes to participate" (caregiver of patient with IPF) [13] "Even showering becomes a problem; you do it in stages. And someone has to be here" (patient with IPF) [18] "... just the inconvenience of it all loxygen therapyl and the stupid line all over the house, and I trip on, because it's always - my leash, as my husband calls it" (patient with IPF using oxygen for 9-12 months) [26]

"If there was a plan, if we knew what was going on, then we could make decisions.... There are no standard procedures for end-of-life decision makers" (advocate) [15]

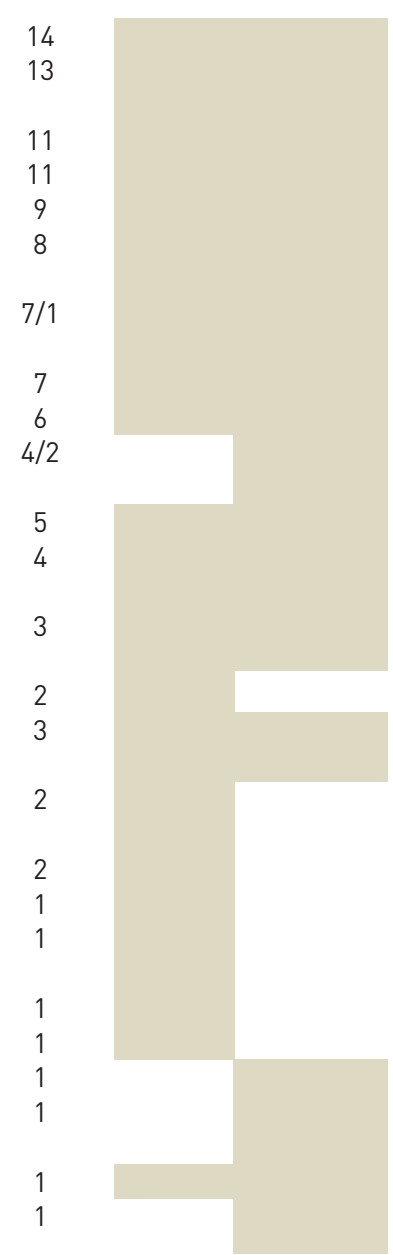

"I am dissatisfied; the disease was not explained to me. I feel like people assumed I know, but in general it felt like you were left, like, in the corner" (non-IPF patient) [21]

"I remember searching on the Internet, and thinking, 'I'll probably be dead next week.' In the beginning, because you know so very little, it can be very frightening. It was so confusing" (patient with IPF) [31]

"They say you are like this and that's how you are going to end up like. And you think, what's going to happen in between?' (patient with IPF) [34]

"[W]hen I went to see him a month or two back, he said, 'Are you using your oxygen?' I said, 'No'. I said, 'It's too bloody hard to connect'.... He said, 'It's the easiest thing you can do. Why haven't you tried it?' I said, 'No one has told me how easy it is"' ( patient with IPF who had ceased antifibrotic therapyl [16]

"[l]t would be wonderful to have, once-a-month, a highlight on somebody who says "Yeah, I've been dealing with this for 11 years, this is what my life is like" instead of reading some statistic" (patient with IPF) [23]

"It is difficult for us to help them, we would like to have more information about what we should do at home" (caregiver of patient with IPF using pirfenidonel [8] 
TABLE 4 Summary of supportive care needs; health system/communication and access to care domains

Needs domain and category
Studies $n$

Individual reporting need

\section{Representative quotes}

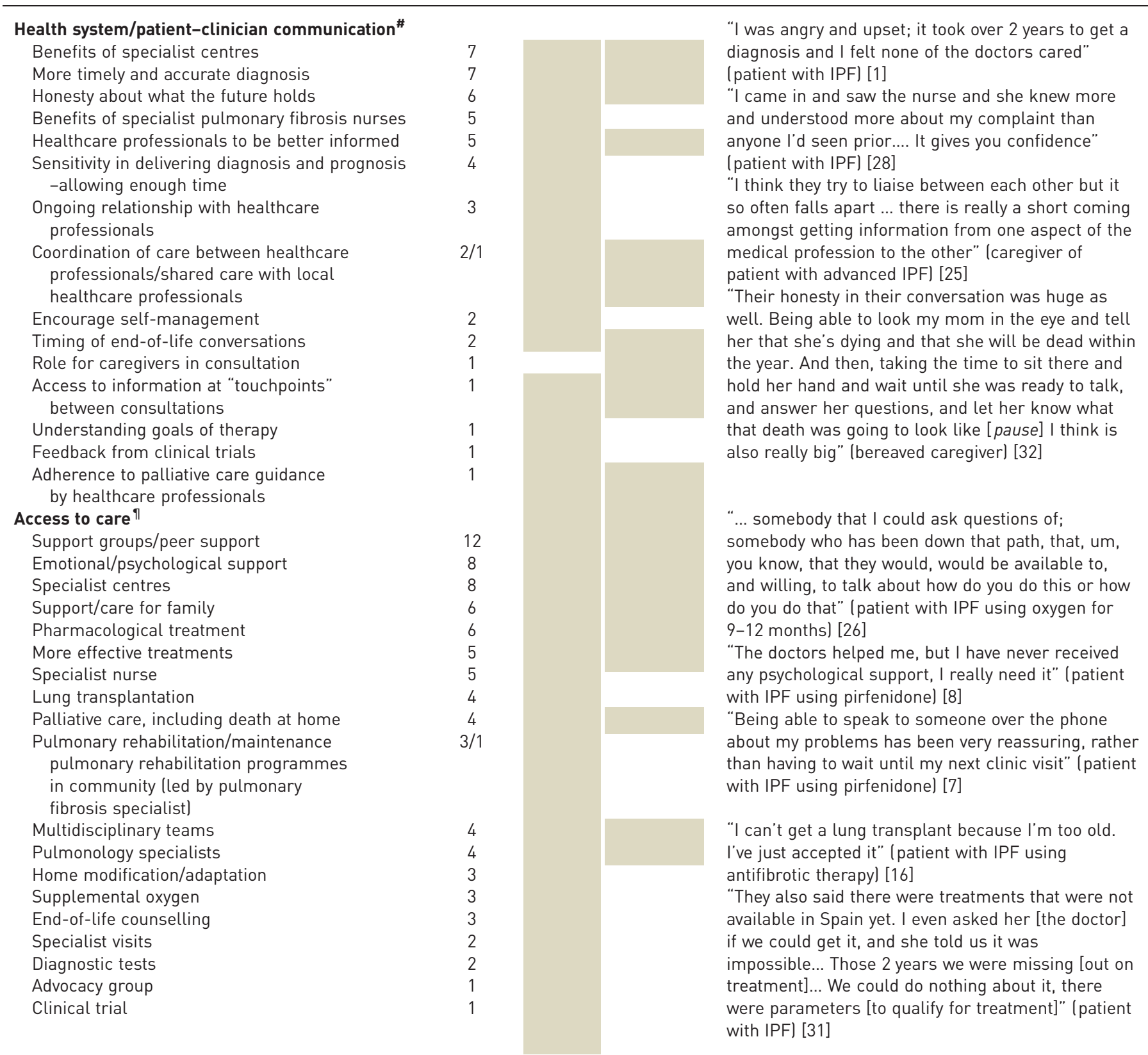

Shading indicates where supportive care need was identified by the patient and/or caregiver. IPF: idiopathic pulmonary fibrosis. ${ }^{\text {\# }} \mathrm{n}=22$; ๆ: $n=27$.

\section{Practical/daily living needs}

The practical/daily living needs of patients and caregivers were reported in 18 studies (table 3 ). The ability of patients to perform activities of daily living diminished over time, including tasks such as shaving, showering and shopping $[18,27,28]$. Many had given up hobbies and activities that they once enjoyed [3, $13,18,26,30]$. Distress related to loss of independence and inability to complete simple daily tasks was reported by $18 \%$ of patients ( $95 \%$ CI $10-26 \%$ ) [39]. Practical limitations were particularly obvious among patients who used supplemental oxygen. Caregivers viewed patients as being "tied" to their oxygen [19]. 
The challenge of transporting the oxygen tank required careful planning of excursions [16, 19]. Caregivers assumed physical duties related to oxygen use, such as filling tanks and cleaning the equipment [13, 19]. Both patients and caregivers wanted practical assistance with end-of-life planning [38]. Although discussion of this topic was less acceptable in some countries, patients generally wanted to know more about the dying process and whether their symptoms would be controlled towards the end of life; many expressed that they would like to be prepared and have their "affairs in order" [27].

\section{Information/education needs}

The information/education needs of patients and caregivers were reported in 26 studies (table 3). Over half of the patients and caregivers reported that there was a lack of information and resources about pulmonary fibrosis at the time of diagnosis [17]. The majority of patients (77\%) had not heard of pulmonary fibrosis when they were diagnosed and only $57 \%$ remembered being told that IPF is progressive at the time of diagnosis, while $43 \%$ remembered being informed about treatment options [31]. The most common topics for which practical information was desired were disease progression and prognosis, how to manage medications and their side-effects use of supplemental oxygen, end-of-life planning and the dying process, and practical coping strategies for living with pulmonary fibrosis $[1,3,7$, $8,12,15-17,19-21,23,26-30,40]$. Both patients and caregivers also desired more information on: how to manage breathlessness and cough [7, 12, 21, 29, 30, 37, 40]; the basic mechanisms and pathophysiology of pulmonary fibrosis $[3,8,12,21,24,40]$; nonpharmacological therapies such as exercise and diet $[7,8,12$, $17,30]$; current research and feedback from clinical trials $[8,12,16,23,28]$; interpretation of clinical tests used for diagnosis and monitoring [7, 12, 21, 30]; and how to access treatment centres and referrals [12, 24]. Both patients and caregivers wanted to learn how to recognise and deal with important signs and symptoms $[16,23]$, while caregivers wanted more information on emotional and practical care of their loved ones, including how to avoid infections $[8,23,30]$.

\section{Health system/patient-clinician communication}

The health system/patient-clinician needs of patients and caregivers were reported in 22 studies (table 4). Patients and caregivers identified a lack of awareness of pulmonary fibrosis among health professionals, leading to delays in obtaining a diagnosis $[1,14,21]$. It was important that the diagnosis and prognosis were delivered in a "sensitive and unhurried manner", allowing the patients to express concerns and digest the information $[1,18,23]$. Most believed early referral to a specialist centre was beneficial for timely diagnosis, access to clinical trials, access to medications and obtaining trustworthy information $[14,16,18$, 20, 24, 28, 30]. However, in a European study, one-third of patients preferred that their care was shared between the expert centre and their local pulmonologist [24]. They preferred health professionals to be honest about the prognosis and give information on what to expect over the course of the disease; however, some perceived that this information was withheld $[20,21,29,30]$. Some patients expressed concerns about the timing of end-of-life planning discussions and felt that care should be taken by health professionals when initiating these difficult conversations [3]. Patients found the specialist pulmonary fibrosis nurse to be an important source of information and support [24]. The opportunity to access information at "touchpoints" between consultations was highly valued, as was independent advice for caregivers [30]. Patients wanted their health professionals to provide more opportunities for self-management, as it gave hope and a sense of control [16]. They wanted different health professionals to work together to lessen the burden of medication side-effects and reduce the chance of scheduling appointments at conflicting times.

\section{Access to care}

The access to care of patients and caregivers were reported in 27 studies (table 4). Both patients and caregivers wanted better access to peer support $[12,14,16,18,20,22,26,29,30,40]$. They wanted access to counselling or psychological support services from the point of receiving a diagnosis to the end of the pulmonary fibrosis journey $[1,8,14,15,18,21,22]$. Patients expressed concerns for the support needs of their families $[8,14,24,37]$. Many patients also identified a need for patient-based advocacy groups for ongoing education and support [1]; however, such services were often perceived as lacking [14, 21, 24]. Although patients noted that pulmonary rehabilitation provided valuable emotional support [14], it was not always available, accessible or affordable $[14,16]$. Patients perceived that there were insufficient specialist centres, respiratory specialists and specialist nurses, especially in regional areas $[16,20,24]$. The vast majority of patients in a European study thought seeing a pulmonary fibrosis specialist nurse was beneficial $(88 \%)$ and $21 \%$ would like to see the pulmonary fibrosis nurse every time they visited their doctor, but in some countries such nurses were not available [24]. Due to the unpredictability and complexity of pulmonary fibrosis, patients believed specialist visits should be more frequent, and that early multidisciplinary support should be incorporated $[1,14,30]$. Patients sometimes struggled to afford antifibrotic drugs, supplemental oxygen, diagnostic tests, respiratory seminars and home modifications [14, 
16, 20]. Patients perceived that lung transplantation was frequently inaccessible due to criteria for candidate selection and/or lack of available organs [14].

Planned subgroup analyses

IPF versus other types of pulmonary fibrosis

The vast majority (96\%) of participants were patients with IPF or their caregivers, so there was insufficient data to determine whether supportive care needs were different for other pulmonary fibrosis diagnoses.

Antifibrotic era versus pre-antifibrotic era

In 17 studies it was possible to confirm that the data had been collected in the antifibrotic era $[7,8,14,16$, 20-24, 30-35, 41, 42]. These studies identified supportive care needs across all nine domains. In the physical/cognitive domain these studies were more heavily represented than studies from the pre-antifibrotic era in the theme of managing treatment side-effects (gastrointestinal, photosensitivity and weight loss). In the health system domain, these studies were more heavily represented in themes related to the benefits of specialist pulmonary fibrosis centres and specialist pulmonary fibrosis nurses. In the access to care domain, these studies were the sole contributors to the theme of difficulty accessing pharmacological treatment, either because the antifibrotic therapy was not approved or too expensive.

Disease stage

No studies reported patient needs at the time of diagnosis separately to those during treatment and follow-up. Six studies specifically addressed end-of-life care $[3,20,25,32,37,40]$, reporting supportive care needs in all nine domains, with no evidence that themes differed from the other studies. The need for information on end-of-life planning was also identified by patients and caregivers in six additional studies that addressed more general aspects of treatment (information domain) $[15,21,27,29,30,33]$, suggesting this need is not restricted to those nearing the end-of-life.

\section{Discussion}

This systematic review shows that people with pulmonary fibrosis have important supportive care needs across a wide range of domains. The most frequently reported needs were in the domains of information/ education, particularly at the time of diagnosis, as well as psychosocial/emotional support. Psychosocial needs were evident across both patients and their caregivers, including help with managing anxiety, fear, anger, sadness and grief. Practical needs for support with activities of daily living were also expressed by both patients and caregivers, including loss of independence, financial burden and challenges managing oxygen equipment. Importantly, the review identified an additional domain of "access to care", including access to peer support, psychological support, specialist centres and family support; these challenges were reported across many different countries and health systems.

The supportive care needs of people with pulmonary fibrosis do not appear to have changed substantially in the antifibrotic era, aside from a greater need to manage drug side-effects and increased awareness of the benefits of specialist pulmonary fibrosis services. Therefore, it seems likely that advances in supportive care have not kept pace with advances in other aspects of treatment. For instance, while anxiety and depression have been thoroughly documented in people with pulmonary fibrosis in recent years, specific treatment strategies for this group are lacking [43]. The enormous psychosocial and emotional burden of pulmonary fibrosis documented in this review suggests that this should be a priority for clinical practice and future research. The desire for peer support was particularly evident, with many patients and caregivers expressing their desire for support groups and increased psychosocial care. Patient advocacy organisations frequently have a critical role in providing supportive care services, with pharmaceutical companies also active in this area [7]. However study participants had a clear expectation that such services would also be linked with their healthcare team (table 4) and emphasised the important role of specialist pulmonary fibrosis centres. These data suggest that supportive care needs could be addressed by multidisciplinary pulmonary fibrosis healthcare teams that are well integrated with other health, community and social care services.

While some of the supportive care needs related directly to health and wellbeing, others related to the broader social context. Stigma and lack of community understanding of pulmonary fibrosis were frequently reported (table 2), which added to patient distress. Health-related stigma has been defined as an adverse social judgement based on an enduring feature of identity conferred by a health problem [44]. Stigma is commonly reported across a range of chronic lung diseases and has adverse impacts on quality of life, psychosocial and physical wellbeing, and experience of treatment [45]. Stigma may therefore impact on other domains identified in this review, including social isolation and access to care. The connection between stigma and other domains of patient experience suggests that the need for information and 
education about pulmonary fibrosis may extend beyond the patient and caregiver (table 3) to health professionals and to the wider community.

A strength of this review is the synthesis of studies conducted across a wide range of settings to provide a comprehensive overview of supportive care needs in pulmonary fibrosis. These data can be used by pulmonary fibrosis service providers to inform the design of care models that directly address the needs of patients and caregivers. Limitations to the review include a lack of included studies addressing the supportive care needs of people with non-IPF diagnoses, which may differ due to the diversity of disease course and prognosis. Most studies used qualitative methodology, which did not allow us to document the prevalence of each supportive care need; however, such methodology provides rich data regarding patient and caregiver experiences.

In conclusion, people with pulmonary fibrosis and their caregivers have a wide range of supportive care needs, particularly for increased information about their disease and its treatment, and better psychosocial and emotional support. This provides opportunities to optimise delivery of comprehensive pulmonary fibrosis care to meet the needs of people living with pulmonary fibrosis and those who care for them.

Conflict of interest: J.Y.T. Lee has nothing to disclose. G. Tikellis has nothing to disclose. T.J. Corte reports grants and personal fees from Roche and Boehringer, and grants from Galapagos, Actelion and Sanofi, outside the submitted work. N.S. Goh has nothing to disclose. G.I. Keir has nothing to disclose. L. Spencer has nothing to disclose. D. Sandford has nothing to disclose. Y.H. Khor reports non-financial support from Air Liquide Healthcare, grants and personal fees from Boehringer Ingelheim, and personal fees from Roche, outside the submitted work. I. Glaspole reports personal fees from Boehringer Ingelheim, Roche, Menarini, Pulmotect and Avalyn, outside the submitted work. J. Price has nothing to disclose. A.J. Hey-Cunningham has nothing to disclose. J. Maloney reports unrestricted educational grants to her institution (Lung Foundation Australia) from Boehringer Ingelheim and Roche Australia, outside the submitted work. A.K. Teoh has nothing to disclose. A.L. Watson has nothing to disclose. A.E. Holland reports grants from National Health and Medical Research Council Australia, during the conduct of the study; and unrestricted research grants from Boehringer Ingelheim and Roche, outside the submitted work.

Support statement: This study was funded by the Centre of Research Excellence in Pulmonary Fibrosis (funded by the National Health and Medical Council (Australia); GNT1116371) and supported by Foundation partner Boehringer Ingelheim and Program Partners Roche and Galapagos. Funding information for this article has been deposited with the Crossref Funder Registry.

\section{References}

1 Schoenheit G, Becattelli I, Cohen AH. Living with idiopathic pulmonary fibrosis: an in-depth qualitative survey of European patients. Chron Respir Dis 2011; 8: 225-231.

2 Bajwah S, Ross JR, Peacock JL, et al. Interventions to improve symptoms and quality of life of patients with fibrotic interstitial lung disease: a systematic review of the literature. Thorax 2013; 68: 867-879.

3 Bajwah S, Higginson IJ, Ross JR, et al. The palliative care needs for fibrotic interstitial lung disease: a qualitative study of patients, informal caregivers and health professionals. Palliat Med 2013; 27: 869-876.

4 Carvajalino S, Reigada C, Johnson MJ, et al. Symptom prevalence of patients with fibrotic interstitial lung disease: a systematic literature review. BMC Pulm Med 2018; 18: 78.

5 Holland AE, Fiore JF Jr., Bell EC, et al. Dyspnoea and comorbidity contribute to anxiety and depression in interstitial lung disease. Respirology 2014; 19: 1215-1221.

6 Hui D. Definition of supportive care: does the semantic matter? Curr Opin Oncol 2014; 26: 372-379.

7 Duck A, Pigram L, Errhalt P, et al. IPF care: a support program for patients with idiopathic pulmonary fibrosis treated with pirfenidone in Europe. Adv Ther 2015; 32: 87-107.

8 Russell AM, Ripamonti E, Vancheri C. Qualitative European survey of patients with idiopathic pulmonary fibrosis: patients' perspectives of the disease and treatment. BMC Pulm Med 2016; 16: 10 .

9 Moher D, Liberati A, Tetzlaff J, et al. Preferred reporting items for systematic reviews and meta-analyses: the PRISMA statement. BMJ 2009; 339: b2535.

10 Kmet LM, Lee RC, Cook LS. Standard quality assessment criteria for evaluating primary research papers from a variety of fields. Alberta, Alberta Heritage Foundation for Medical Research, 2004.

11 Kotronoulas G, Papadopoulou C, Burns-Cunningham K, et al. A systematic review of the supportive care needs of people living with and beyond cancer of the colon and/or rectum. Eur J Oncol Nurs 2017; 29: 60-70.

12 Albright K, Walker T, Baird S, et al. Seeking and sharing: why the pulmonary fibrosis community engages the web 2.0 environment. BMC Pulm Med 2016; 16: 4.

13 Belkin A, Albright K, Swigris JJ. A qualitative study of informal caregivers' perspectives on the effects of idiopathic pulmonary fibrosis. BMJ Open Respir Res 2014; 1: e000007.

14 Bonella F, Wijsenbeek M, Molina-Molina M, et al. European IPF Patient Charter: unmet needs and a call to action for healthcare policymakers. Eur Respir J 2016; 47: 597-606.

15 Bridges JFP, Paly VF, Barker E, et al. Identifying the benefits and risks of emerging treatments for idiopathic pulmonary fibrosis: a qualitative study. Patient 2014; 8: 85-92.

16 Burnett K, Glaspole I, Holland AE. Understanding the patient's experience of care in idiopathic pulmonary fibrosis. Respirology 2019; 24: 270-277.

17 Collard HR, Tino G, Noble PW, et al. Patient experiences with pulmonary fibrosis. Respir Med 2007; 101: $1350-1354$

18 Giot C, Maronati M, Becattelli I, et al. Idiopathic pulmonary fibrosis: an EU patient perspective survey. Curr Respir Med Rev 2013; 9: 112-119. 
19 Graney BA, Wamboldt FS, Baird S, et al. Informal caregivers experience of supplemental oxygen in pulmonary fibrosis. Health Qual Life Outcomes 2017; 15: 133.

20 Lindell KO, Kavalieratos D, Gibson KF, et al. The palliative care needs of patients with idiopathic pulmonary fibrosis: a qualitative study of patients and family caregivers. Heart Lung 2017; 46: 24-29.

21 Morisset J, Dubé B-P, Garvey C, et al. The unmet educational needs of patients with interstitial lung disease. Setting the stage for tailored pulmonary rehabilitation. Ann Am Thorac Soc 2016; 13: 1026-1033.

22 Overgaard D, Kaldan G, Marsaa K, et al. The lived experience with idiopathic pulmonary fibrosis: a qualitative study. Eur Respir J 2016; 47: 1472-1480.

23 Ramadurai D, Corder S, Churney T, et al. Understanding the informational needs of patients with IPF and their caregivers: "You get diagnosed, and you ask this question right away, what does this mean?". BMJ Open Qual 2018; 7: e000207.

24 van Manen MJG, Kreuter M, van den Blink B, et al. What patients with pulmonary fibrosis and their partners think: a live, educative survey in the Netherlands and Germany. ERJ Open Res 2017; 3: 00065-2016.

25 Bajwah S, Koffman J, Higginson IJ, et al. 'I wish I knew more ...' the end-of-life planning and information needs for end-stage fibrotic interstitial lung disease: views of patients, carers and health professionals. BMJ Support Palliat Care 2013; 3: 84-90.

26 Graney BA, Wamboldt FS, Baird S, et al. Looking ahead and behind at supplemental oxygen: a qualitative study of patients with pulmonary fibrosis. Heart Lung 2017; 46: 387-393.

27 Swigris JJ, Stewart AL, Gould MK, et al. Patients' perspectives on how idiopathic pulmonary fibrosis affects the quality of their lives. Health Qual Life Outcomes 2005; 3: 61.

28 Duck A, Spencer LG, Bailey S, et al. Perceptions, experiences and needs of patients with idiopathic pulmonary fibrosis. J Adv Nurs 2015; 71: 1055-1065.

29 Holland AE, Fiore JF Jr., Goh N, et al. Be honest and help me prepare for the future: what people with interstitial lung disease want from education in pulmonary rehabilitation. Chron Respir Dis 2015; 12: 93-101.

30 Sampson C, Gill BH, Harrison NK, et al. The care needs of patients with idiopathic pulmonary fibrosis and their carers (CaNoPy): results of a qualitative study. BMC Pulm Med 2015; 15: 155.

31 Maher T, Swigris JJ, Kreuter M, et al. Identifying barriers to idiopathic pulmonary fibrosis treatment: a survey of patient and physician views. Respiration 2018; 96: 514-524.

32 Pooler C, Richman-Eisenstat J, Kalluri M. Early integrated palliative approach for idiopathic pulmonary fibrosis: a narrative study of bereaved caregivers' experiences. Palliat Med 2018; 32: 1455-1464.

33 Ramadurai $\mathrm{D}$, Corder S, Churney $\mathrm{T}$, et al. Idiopathic pulmonary fibrosis: educational needs of health-care providers, patients, and caregivers. Chron Respir Dis 2019; 16: 1-8.

34 Senanayake S, Harrison K, Lewis M, et al. Patients' experiences of coping with idiopathic pulmonary fibrosis and their recommendations for its clinical management. PLoS One 2018; 13: e0197660.

35 Shah RJ, Collard HR, Morisset J. Burden, resilience and coping in caregivers of patients with interstitial lung disease. Heart Lung 2018; 47: 264-268.

36 Conoscenti CS, Rubin EM, Sapiro N. Patient journey with idiopathic pulmonary fibrosis (IPF): a breathtaking experience. Am J Respir Crit Care Med 2013; 187: A1090.

37 Gillon S, Sutherland T, Slough J. Improving palliative care for patients with idiopathic pulmonary fibrosis. Palliat Med 2016; 30: Poster 189.

38 Killin CR, Hayes J, Byrne A, et al. Quality of care for patients with idiopathic pulmonary fibrosis - perspectives from patients and carers. Palliat Med 2010; 24: S178-S179.

39 Wall J, Crosby V, Hussain A, et al. Establishing the palliative and supportive care needs of patients with idiopathic pulmonary fibrosis and non specific interstitial pneumonia. Thorax 2013; 68: A165-A1A6.

40 Wright J, Cove J, Russell AM, et al. Pilot study to test the feasibility of introducing palliative care as part of a psychological support workshop for patients newly diagnosed with idiopathic pulmonary fibrosis (IPF) and their families. Palliat Med 2016; 30: P324.

41 Belz A, Debowska P, Warzecha J, et al. Patients' expectations and quality of life before introduction of pirfenidone used in idiopathic pulmonary fibrosis. Eur Resp J 2018; 52: Suppl. 62, PA4784.

42 McLean A, Webster S, Fry M, et al. Priorities and expectations of patients attending a multidisciplinary interstitial lung disease clinic. Respirology 2018; 23: 146.

43 Jo HE, Prasad JD, Troy LK, et al. Diagnosis and management of idiopathic pulmonary fibrosis: Thoracic society of Australia and New Zealand and Lung Foundation Australia position statements summary. Med J Aust 2018; 208: 82-88.

44 Weiss MG, Ramakrishna J, Somma D. Health-related stigma: rethinking concepts and interventions. Psychol Health Med 2006; 11: 277-287.

45 Rose S, Paul C, Boyes A, et al. Stigma-related experiences in non-communicable respiratory diseases: a systematic review. Chron Respir Dis 2017; 14: 199-216. 